\title{
||||||||||||||||||||||||||||||||||||||||||||||||||||||||||||||||||||.
}

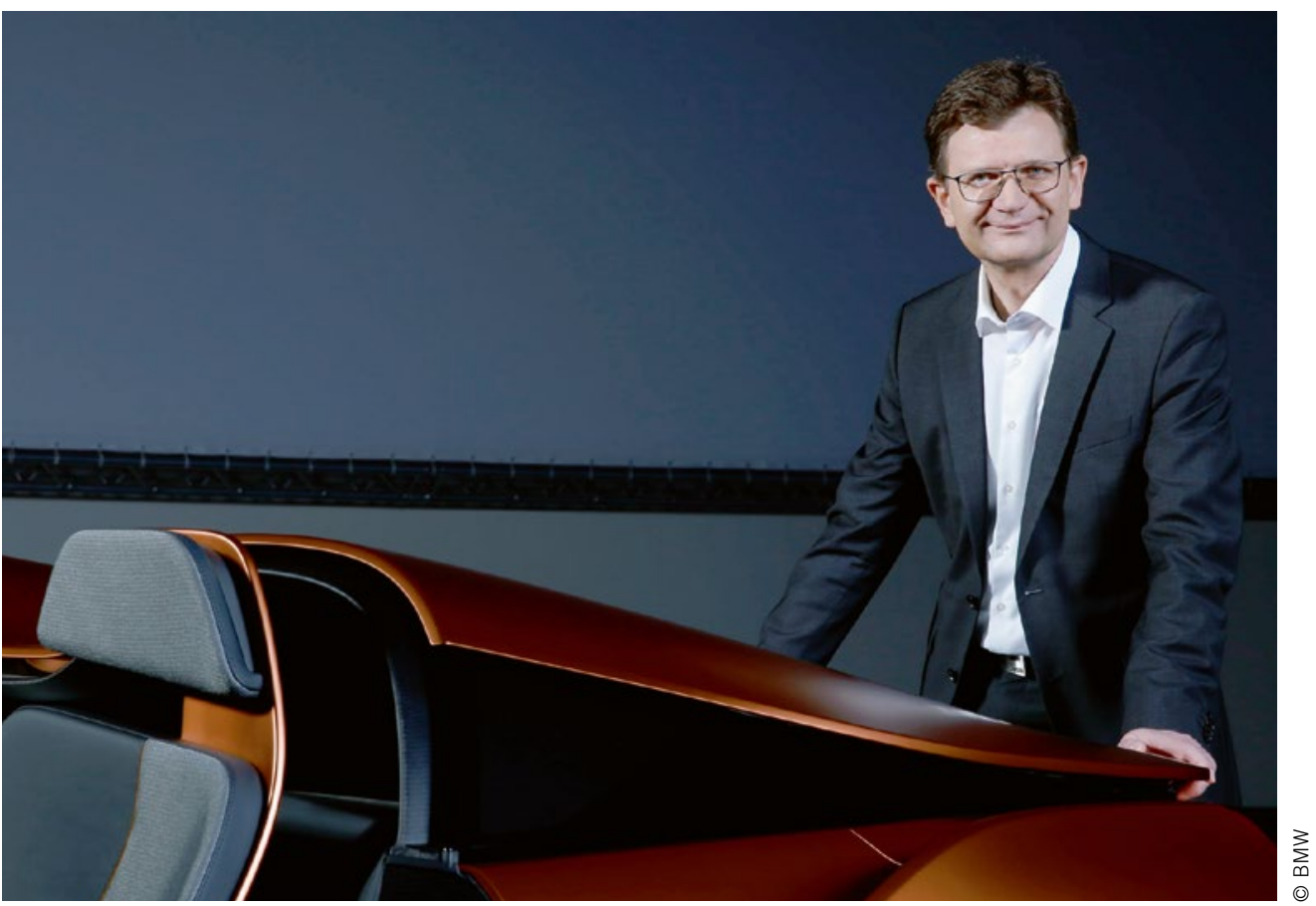

Dipl.-Ing. Klaus Fröhlich Member of the Board of Management of BMW AG, Development, Member of the ATZ Advisory Board

\section{Fully Autonomous, Highly Connected and Emission-free}

In my last guest commentary for ATZ I wrote: "Over the next decade, cars will change more radically than they have in the past fifty years." Two years later, it is clear that the pace of change has increased significantly. In particular in the area of autonomous and highly automated driving, we need development hubs of a kind that would simply not have been possible just a few years ago.

In contrast to what marketing departments may claim, I believe that autonomous driving presents us with an enormous challenge. It involves replacing human sensory perception and the brain's thinking and decision-making powers with an intelligent system.

There are two driving forces behind the autonomous vehicle trend. One is the fact that customers do not always want to drive themselves. The other is new business models. At the BMW Group, we believe that drivers should decide according to the situation. They can either drive themselves in a vehicle fully customised to meet their requirements, where everything revolves around them as the "Ultimate Driver". We call this Boost Mode. Or in the near future they will have the option of fully automated driving with all its benefits from relaxation to entertainment, which we describe as Ease Mode.
However, as an individual car manufacturer we will not be able to overcome this challenge alone. The future of the automotive industry depends on the general conditions and, most importantly, on our willingness to cooperate. We have clearly defined the areas where we need to enter into partnerships. We and other carmakers have joined together to buy Here in order to ensure that digital maps will be available to all the players on the market. This mapping service gives us access to highresolution maps, which alongside the sensor systems in cars are the other key requirement for autonomous driving. We are now working with our partners Intel, Mobileye, Delphi and Continental on industrialising the sensor technology and the information processing systems in cars. Something that we are already independently very good at is motion control, in other words, designing and controlling cars using the drive system, the chassis and the brakes, and also complete system integration.

With the BMW iNext project, we have committed to introducing fully autonomous driving by 2021 . The iNext will, of course, also be emission-free. But for us that on its own no longer represents innovation. With fully autonomous driving capability, comprehensive connectivity and the interior of the future, the Project i 2.0 will act as the incubator for our brand's innovative capacity. 